\title{
Holographic RG flow triggered by a classically marginal operator
}

\author{
Chanyong Park $\odot^{*}$ \\ Department of Physics and Photon Science, Gwangju Institute of Science and Technology, \\ Gwangju 61005, South Korea
}

(Received 16 September 2021; accepted 23 January 2022; published 4 February 2022)

\begin{abstract}
We study a holographic renormalization group $(\mathrm{RG})$ flow triggered by a classically marginal operator. When a marginal operator deforms a conformal field theory, it does not yield a nontrivial RG flow at the classical level. At the quantum level, however, quantum corrections modify a marginal operator into one of the truly marginal, marginally relevant, and marginally irrelevant operators. This quantum correction may generate a nontrivial RG flow. We investigate how we can describe such an RG flow holographically with quantum corrections. Applying the holographic technique, we look into the change of physical quantities, like a coupling constant and vacuum expectation value, along the RG flow. We also study the holographic description of the trace anomaly caused by the gluon condensation.
\end{abstract}

DOI: 10.1103/PhysRevD.105.046004

\section{INTRODUCTION}

For the last decade, much attention has been paid to understanding strongly interacting systems of QCD and condensed matter theory by applying the AdS/CFT correspondence [1-4]. The AdS/CFT correspondence has passed many nontrivial checks. When we take into account a relevant deformation, the original conformal field theory (CFT) changes along with an energy scale observing the system and eventually flows to a new IR theory [5]. At a low-energy scale, nuclear and condensed matter physics usually have a strong coupling constant. Understanding such nonperturbative physics governed by strong interactions is one of the important issues to be resolved in theoretical and experimental researches. To account for nonperturbative phenomena, we need to figure out an exact and nonperturbative renormalization group (RG) flow. Although the perturbative RG flow was well established in quantum field theory (QFT), it is not the case for the nonperturbative RG flow. In this situation, the AdS/CFT correspondence, or holography, can shed light on this issue due to the relation between the nonperturbative RG flow of QFT and the classical gravity theory [6-20]. In this work, we investigate a holographic RG flow triggered by a marginal deformation and compare the holographic result with the gluon condensation of the lattice QCD [21,22].

\section{*yong21@gist.ac.kr}

Published by the American Physical Society under the terms of the Creative Commons Attribution 4.0 International license. Further distribution of this work must maintain attribution to the author(s) and the published article's title, journal citation, and DOI. Funded by SCOAP.
After the AdS/CFT conjecture, there were many attempts to understand the dual QFT of an asymptotic anti-de Sitter (AdS) geometry. In the holographic setup, an IR scale of gravity theory maps to a UV scale of dual field theory and generally leads to various UV divergences of a dual QFT [9-11,13]. In order to get rid of such UV divergences, several distinct methods have been invented. One of them is the 'background subtraction' method, which gets rid of the effect of the background reference spacetime. This subtraction method is not applicable to certain cases where an appropriate reference solution is ambiguous or unknown, e.g., topological black holes [23-27], TaubNUT-AdS, and Taub-bolt-AdS [28-30]. Another way to remove the UV divergence is called the holographic renormalization in which UV divergences are removed by adding appropriate counterterms. The holographic renormalization method is similar to the renormalization scheme of a QFT, and useful in that it does not require a background reference spacetime [10]. Due to this similarity, the holographic RG flow can be regarded as the Wilsonian RG flow of the dual QFT [5]. There exists another description called the RG flow of the entanglement entropy where the RG scale is characterized by the subsystem size. It has been shown that the thermodynamicslike law of the entanglement entropy can reproduce the linearized Einstein equation of the dual AdS geometry [31-33]. From the field theory point of view, the RG flow of the entanglement entropy corresponds to the real space RG flow [34-40]. For the entanglement entropy RG flow, intriguingly, it was shown that a quantum entanglement entropy in the UV region can flow to a thermal entropy in the IR region [41].

When a CFT deforms by a marginal operator, the deformed theory remains conformal at the classical or 
action level. However, this is not true at the quantum level because quantum corrections may change the property of the deformation operator. The AdS/CFT correspondence claimed that a classical gravity theory is dual to a full quantum field theory defined at the boundary. Therefore, the holographic description allows us to figure out the nonperturbative RG flow including all quantum corrections. One of the important quantities affected by such quantum corrections is a $\beta$-function. A $\beta$-function describes how a coupling constant depends on the energy scale. In general, physical phenomena crucially depend on the strength of a coupling constant. At the classical level, a $\beta$-function is classified by the conformal dimension of a deformation operator. When a coupling constant $\lambda$ couples to an operator with a conformal dimension $\Delta$ in a $d$-dimensional CFT, a classical $\beta$-function is given by [42]

$$
\beta_{c l}=-(d-\Delta) \lambda .
$$

If we further take into account quantum corrections, the $\beta$-function near the fixed point is generalized to

$$
\beta=\beta_{c l}+\beta_{q},
$$

where $\beta_{q}$ indicates quantum corrections. If we concentrate on a classically marginal operator with $\Delta=d$, the classical $\beta$-function automatically vanishes. However, the quantum effect leads to a nonvanishing $\beta$-function and generates a nontrivial RG flow. As explained here, the quantum correction plays a crucial role in determining the RG flow triggered by a marginal operator. In this work, we investigate the holographic description of such quantum corrections and discuss how a marginal deformation modifies a UV CFT. This is a general feature of the RG flow of a marginal operator.

The gluon condensation, $\langle G\rangle$, in QCD is one of the typical examples for a classically marginal deformation which is important to characterize a nonperturbative ground state. At a tree level, the gluon condensation is trivial because it is marginal. However, its one-loop correction in the UV region leads to the following relation

$$
\left\langle T_{\mu}^{\mu}\right\rangle=-\frac{N_{c}}{8 \pi} \frac{\beta_{\lambda}}{\lambda^{2}}\langle G\rangle,
$$

where $\lambda$ and $\beta_{\lambda}$ indicate the 't Hooft coupling constant and its $\beta$-function, respectively. This relation was called the trace anomaly $[21,22]$. This relation indicates that the trace anomaly is crucially associated with the RG flow caused by the gluon condensation. In the present work, we investigate how to describe the trace anomaly in terms of the holographic RG flow. According to the AdS/CFT correspondence, the holographic description corresponds to the nonperturbative description of the dual QFT. Therefore, the correct description of the holographic RG flow can shed light on understanding the nonperturbative RG flow of QFTs, QCD and condensed matter theory.

The rest of this paper is organized as follows. In Sec. II we discuss a $\beta$-function of a marginal operator and an RG equation appearing of a traditional QFT. In Sec. III we study a gravity theory involving a scalar field which is dual to a marginal operator. Moreover, we investigate how to construct the holographic RG flow triggered by such a marginal operator. By applying this holographic RG flow, we look into the trace anomaly caused by the gluon condensation in Sec. IV. Lastly, we finish this work with some concluding remarks in Sec. V.

\section{RG FLOW IN QFT}

At a low-energy scale, understanding new physics of macroscopic systems is one of the important issues to be resolved. To understand macroscopic theories, we need to know how a microscopic UV theory flows to a new IR theory along the RG flow. A relevant or marginally relevant operator causes a nontrivial RG flow and its effect becomes significant in the IR region. In this RG flow procedure, nonperturbative quantum corrections play a crucial role. Unfortunately, we do not have an analytic method accounting for such a nonperturbative RG flow in a traditional QFT. In this situation, the AdS/CFT correspondence may provide a new chance to figure out a nonperturbative RG flow.

For later comparison with the holographic result, we first discuss general aspects of the RG flow in a $d$-dimensional QFT. Assuming that a Euclidean CFT deforms by an operator of a conformal dimension $\Delta$, the deformed theory is described by

$$
S_{\mathrm{QFT}}=S_{\mathrm{CFT}}+\int d^{d} x \sqrt{\gamma} \mu^{d-\Delta} \lambda \bar{O},
$$

where $\gamma_{\mu \nu}$ corresponds to a background metric and $\bar{O}$ is a dimensionful composite operator of fundamental fields. Here, $\mu$ and $\lambda$ denote an RG scale and dimensionless coupling, respectively. Ignoring quantum effects, the deformation can be characterized by the conformal dimension $\Delta$. At the classical level, for example, the dimensionless coupling constant and dimensionless operator, $O=\mu^{-\Delta} \bar{O}$, under the RG flow transform as $[5,42]$

$$
\lambda \rightarrow \mu^{-(d-\Delta)} \lambda \quad \text { and } \quad O \rightarrow \mu^{-\Delta} O .
$$

Under this RG transformation, a deformation operator is classified into three different categories; relevant for $\Delta<d$, marginal for $\Delta=d$, and irrelevant for $\Delta>d$. The deformation effect of a relevant operator is negligible in the UV regime, while it becomes important in the IR regime and changes the UV theory into another IR theory. A marginal operator at the classical level does not break the conformal symmetry, so that the deformed theory remains conformal 
in the entire energy scale. The irrelevant operator gives rise to a serious effect on the UV theory, so that the resulting theory even in the UV limit differs from the original CFT. This means that the deformed theory is UV incomplete. These features become manifest when we take into account a classical $\beta$-function [42]

$$
\beta_{c l} \equiv \frac{\partial \lambda}{\partial \log \mu}=-(d-\Delta) \lambda .
$$

A solution of the classical $\beta$-function automatically satisfies the scaling behavior in (2.2). For a marginal operator with $\Delta=d$, the classical $\beta$-function automatically vanishes and the coupling constant does not change along the RG flow. The coupling constant of an irrelevant operator has a positive $\beta$-function, so that the coupling constant becomes large as $\mu$ increases. This implies that the effect of the irrelevant deformation becomes more important in the UV region. As a consequence, the deformed theory is not the same as the undeformed one even in the UV limit. On the other hand, a relevant operator has a negative $\beta$-function and its coupling constant decreases as $\mu$ increases. Therefore, the effect of a relevant operator is negligible in the UV region. Note that this is the story at the classical level. If we further take into account quantum effects, they can modify the behavior of the classical $\beta$-function.

Quantum effects in the renormalization procedure usually cause various UV divergences. Since physical quantities must be finite, we need to get rid of such UV divergences by adding appropriate counterterms. After renormalization, the renormalized partition function usually reduces to

$$
\mathcal{Z}=\int \mathcal{D} \phi e^{-\left(S_{\mathrm{QFT}}+S_{c t}\right)}=e^{-\Gamma\left[\gamma_{\mu \nu}(\mu), \lambda(\mu) ; \mu\right]},
$$

where $\Gamma$ is called a generating functional depending on coupling constants. Although the background metric $\gamma_{\mu \nu}$ in a QFT does not vary, in the present work we take into account the background metric as another coupling constant as done in Ref. [43,44]. Since the renormalized partition function must be independent of the RG scale, the variation of the partition function gives rise to the following $\mathrm{RG}$ equation

$$
0=\frac{\mu}{\sqrt{\gamma}} \frac{\partial \Gamma}{\partial \mu}+\gamma^{\mu \nu}\left\langle T_{\mu \nu}\right\rangle+\beta_{\lambda}\langle O\rangle,
$$

where

$$
\begin{gathered}
\beta_{\lambda}=\frac{d \lambda}{d \log \mu}, \\
\left\langle T_{\mu \nu}\right\rangle=-\frac{2}{\sqrt{\gamma}} \frac{\partial \Gamma}{\partial \gamma^{\mu \nu}},
\end{gathered}
$$

$$
\langle O\rangle=\frac{1}{\sqrt{\gamma}} \frac{\partial \Gamma}{\partial \lambda} .
$$

It is worth noting that the generating functional is given by a function of coupling constants at a given energy scale. Therefore, the VEVs, $\langle O\rangle$ and $\left\langle T_{\mu \nu}\right\rangle$, must be determined as functions of coupling constants whose strength crucially relies on the RG scale.

If the background metric is independent of the RG scale, the above $\mathrm{RG}$ equation reduces to

$$
0=\mu \frac{\partial \Gamma}{\partial \mu}+\beta_{\lambda}\langle O\rangle
$$

This is the typical RG equation of a QFT. However, assuming that the metric scales under the RG transformation, the generalized RG equation (2.5) gives us more information about the stress tensor of a system which plays an important role in understanding the connection to the dual gravity, as will be seen later. For the undeformed CFT with $\beta_{\lambda}=0$, the $\mathrm{RG}$ equation reduces to

$$
\left\langle T_{\mu}^{\mu}\right\rangle=-\frac{\mu}{\sqrt{\gamma}} \frac{\partial \Gamma}{\partial \mu} .
$$

The stress tensor of a CFT must be traceless up to a conformal anomaly [45]. Therefore, we may associate the right hand side with a conformal anomaly which depends on the topology of the background spacetime. For a flat spacetime there is no conformal anomaly. From now on, we focus on a QFT defined in a flat spacetime.

Quantum corrections usually modify the classical $\beta$-function. For a marginal and relevant deformation, the $\beta$-function in the UV region is written as

$$
\beta_{\lambda}=\beta_{c l}+\beta_{q}=-(d-\Delta) \lambda+\beta_{q},
$$

where $\beta_{q}$ means all quantum corrections. For a relevant deformation, the quantum effect becomes subdominant in the $\mathrm{UV}$ region. For a marginal deformation with $\Delta=d$, the classical $\beta$-function vanishes and the action still preserves the scale invariance. Therefore, the classical RG flow becomes trivial and the trace of the stress tensor vanishes. However, this is not the case at the quantum level. The quantum effect can allow a nonvanishing $\beta$-function. If $\beta_{q}=0$, for example, the corresponding operator is called a truly marginal operator. In this case, the conformal symmetry of the UV theory preserves along the RG flow even at the quantum level. Therefore, the deformed theory remains as a CFT in the entire RG scale. For $\beta_{q}<0$ (or $\beta_{q}>0$ ), a classically marginal operator changes into a marginally relevant (or marginally irrelevant) operator. A marginally relevant (or irrelevant) operator becomes similar to a relevant (or irrelevant) operator. The quantum 
correction of a marginally relevant operator leads to a nontrivial RG flow which can generate a nonvanishing trace of the stress tensor, as previously mentioned in the trace anomaly.

\section{HOLOGRAPHIC DUAL OF A MARGINAL DEFORMATION}

According to the AdS/CFT correspondence, a $d$-dimensional QFT is dual to a classical $(d+1)$ dimensional gravity theory. In this case, an extra radial direction of a bulk spacetime is identified with an energy scale of a dual QFT. Therefore, we may expect that a gravity theory realizes a RG flow of a dual QFT. If possible, the AdS/CFT correspondence would be helpful to understand a nonperturbative RG flow because the AdS/CFT correspondence claims that a classical gravity theory maps to a QFT involving all quantum effects. Now, we investigate how we can describe an RG flow including all quantum effects on the dual gravity side.

We take into account a four-dimensional CFT deformed by a scalar operator. According to the AdS/CFT correspondence, its dual gravity theory with a Euclidean signature can be described by

$$
\begin{aligned}
S= & -\frac{1}{2 \kappa^{2}} \int d^{5} X \sqrt{g}\left(\mathcal{R}-2 \Lambda-\frac{1}{2} g^{M N} \partial_{M} \phi \partial_{N} \phi-\frac{1}{2} \frac{m^{2}}{R^{2}} \phi^{2}\right) \\
& +\frac{1}{\kappa^{2}} \int_{\partial \mathcal{M}} d^{4} x \sqrt{\gamma} K,
\end{aligned}
$$

where $\Lambda=-6 / R^{2}$ is a cosmological constant with an AdS radius $R$. Note that above the bulk field $\phi$ is dimensionless and that $g_{M N}$ and $\gamma_{\mu \nu}$ indicate bulk and boundary metrics, respectively. The variation of the gravity action usually has a radial derivative of the metric at the boundary, so that the last Gibbons-Hawking term is required to get rid of such a derivative term or to define a Dirichlet boundary condition well.

For $\phi=0$, in particular, an AdS space appears as a geometric solution

$$
d s^{2}=\frac{R^{2}}{z^{2}}\left(d z^{2}+\delta_{i j} d x^{i} d x^{j}\right),
$$

which is a dual of an undeformed CFT with $\phi=0$. When turning a bulk scalar field on, it corresponds to a deformation operator of the dual CFT. In this case, the mass of the bulk field is related to the conformal dimension of the dual operator

$$
\Delta=2+\sqrt{4+m^{2}} .
$$

Near the asymptotic boundary $(z \rightarrow 0)$, the bulk scalar field allows the following perturbative solution

$$
\phi=c_{1} z^{4-\Delta}(1+\cdots)+c_{2} z^{\Delta}(1+\cdots),
$$

where $c_{1}$ and $c_{2}$ are two integral constants.

In the holographic studies, $c_{1}$ and $c_{2}$ for $\Delta>2$ are usually identified with the source (or coupling constant) and VEV of a deformation operator, respectively [2-4]. For later convenience, hereafter, we call this identification a UV identification. Near a UV fixed point, the UV identification reproduces the correct scaling dimension of a dual operator. However, the UV identification becomes unclear in an IR regime due to the mixing of two independent solutions. To define a coupling constant well at the low-energy scale, we exploit a different identification. After we relate the radial position of the boundary to the RG scale of the dual QFT, we identify the boundary value of the dual bulk field, $\phi$, with the dimensionless coupling constant. For the comparison with the UV identification, from now on we call this prescription a new identification. Under this new identification, $c_{1}$ again becomes a dimensionful coupling constant in the UV limit, while the higher-order correction in (3.4) can be regarded as quantum corrections. After the renormalization, as will be seen later, $c_{2}$ reduces to a VEV of the deformation operator. The new identification in the UV limit is perfectly consistent with the UV identification, as it should do. In an intermediate energy scale, however, the new identification is more useful to describe the change of a coupling constant. For example, if we further consider an additional scalar potential, the gravity theory can allow a local minimum at $\phi=\phi_{i r}$ where $d \phi / d z=0$ is automatically satisfied. From the dual QFT viewpoint, this local minimum corresponds to an IR fixed point where a $\beta$-function must vanish. Under the new identification, $d \phi / d z=0$ at the local minimum is naturally reinterpreted as a vanishing $\beta$-function. From now on, we exploit the new identification in order to define a coupling constant.

In QCD, the gluon condensation is a classically marginal deformation with a conformal dimension $\Delta=4$. A marginal deformation can be holographically described by the following Euclidean Einstein-scalar gravity

$$
\begin{aligned}
S= & -\frac{1}{2 \kappa^{2}} \int d^{5} X \sqrt{g}\left(\mathcal{R}-2 \Lambda-\frac{1}{2} g^{M N} \partial_{M} \phi \partial_{N} \phi\right) \\
& +\frac{1}{\kappa^{2}} \int_{\partial \mathcal{M}} d^{4} x \sqrt{\gamma} K .
\end{aligned}
$$

Assuming that the scalar field depends only on the radial coordinate and that the boundary space is flat, the most general metric ansatz preserving the boundary's planar symmetry is given by

$$
d s^{2}=e^{2 A(y)} \delta_{\mu \nu} d x^{\mu} d x^{\nu}+d y^{2} .
$$

The detail of a geometric solution is governed by $\phi$ and $A$ satisfying the following equations of motion 


$$
\begin{aligned}
& 0=24 \dot{A}^{2}-\dot{\phi}^{2}+4 \Lambda, \\
& 0=12 \ddot{A}+24 \dot{A}^{2}+\dot{\phi}^{2}+4 \Lambda, \\
& 0=\ddot{\phi}+4 \dot{A} \dot{\phi},
\end{aligned}
$$

where the dot means a derivative with respect to $y$. The first equation corresponds to a constraint, while the others describe dynamics of $A$ and $\phi$. Note that only two of them are independent. These equations allow the following analytic solution $[46,47]$

$$
\begin{aligned}
\phi & =\phi_{0}+\eta \sqrt{\frac{3}{2}} \log \left(\frac{4 \sqrt{6}-\phi_{1} z^{4} / R^{4}}{4 \sqrt{6}+\phi_{1} z^{4} / R^{4}}\right), \\
e^{2 A(y)} & =\frac{R^{2}}{z^{2}} \sqrt{1-\frac{\eta^{2} \phi_{1}^{2}}{96} \frac{z^{8}}{R^{8}}},
\end{aligned}
$$

with

$$
z=R e^{-y / R}
$$

where $\phi_{0}$ and $\phi_{1}$ are two integral constants. $\phi_{0}$ corresponds to a coupling constant in the UV limit $(y=\infty)$, as mentioned before. The invariance of the gravity action under $\phi \rightarrow-\phi$ allows two different profiles with $\eta= \pm 1$.

Since a classical gravity theory is dual to a full quantum QFT, the classical geometry in (3.10) must provide us information about the quantum effect of the deformation. The inner geometry of (3.10) for $\eta \neq 0$ deviates from AdS space, so that the conformal symmetry breaks down at an intermediate energy scale. This means that the deformation is not truly marginal. In other words, the deformation in (3.10) is either marginally relevant or marginally irrelevant due to nontrivial quantum corrections. This feature is consistent with our new identification discussed before.

There exists another solution which yields a truly marginal deformation. When the scalar field has a constant profile with $\phi_{1}=0$ which is equivalent to take $\eta=0$, its gravitational backreaction is absent. Thus, the AdS metric is still a solution of the deformed theory

$$
\begin{gathered}
\phi=\phi_{0}, \\
A(y)=\frac{y}{R} .
\end{gathered}
$$

This fact implies that the deformed theory is a CFT even at the quantum level. However, the deformed theory is different from the undeformed one because their dual CFTs have different coupling constants, for example, $\phi=0$ for the undeformed theory but $\phi=\phi_{0}$ for the deformed theory. This is a typical feature of a truly marginal deformation which provides a constant shift of a coupling constant with a vanishing $\beta$-function.

\section{A. Holographic description of the RG flow}

The RG equation is usually represented as the first-order differential equations, while the equations of motion of a gravity theory are governed by the second-order differential equations. To derive the RG flow equations from the bulk ones, we need to reformulate the bulk equations in terms of the first-order differential equations. By using the Hamilton-Jacobi formalism, we rewrite the bulk equations as the combination of the first-order differential equations $[10-13,15,16]$. To do so, we first notice that the metric solution in (3.6) is a specific case of the following general metric

$$
d s^{2}=N^{2} d y^{2}+\gamma_{\mu \nu}(x, y) d x^{\mu} d x^{\nu},
$$

where $N$ is a lapse function and $\gamma_{\mu \nu}=e^{2 A(y)} \delta_{\mu \nu}$. Note that the lapse function is nondynamical and that varying the action with respect to the lapse function gives rise to a constraint. Therefore, we can set $N=1$, without loss of generality, after all calculation.

Regarding the radial coordinate $y$ as a Euclidean time, we can rewrite the previous Einstein-scalar gravity action as a functional form of the extrinsic curvature [10-12]

$$
S=\int d^{4} x d y \sqrt{g} \mathcal{L}
$$

with

$\mathcal{L}=\frac{1}{2 \kappa^{2}}\left[N\left(-\mathcal{R}^{(4)}+K_{\mu \nu} K^{\mu \nu}-K^{2}+2 \Lambda\right)+\frac{1}{2 N} \dot{\phi}^{2}\right]$,

where the extrinsic curvature is defined as

$$
K_{\mu \nu}=\frac{1}{2 N} \frac{\partial \gamma_{\mu \nu}}{\partial y} .
$$

Above $\mathcal{R}^{(4)}$ denotes an intrinsic curvature of the boundary spacetime. Since the boundary is flat in our setup, $\mathcal{R}^{(4)}$ automatically vanishes. The canonical momenta of the boundary metric $\gamma_{\mu \nu}$ and scalar field $\phi$ are given by

$$
\begin{aligned}
\pi_{\mu \nu} & \equiv \frac{\partial S}{\partial \dot{\gamma}^{\mu \nu}}=-\frac{1}{2 \kappa^{2}}\left(K_{\mu \nu}-\gamma_{\mu \nu} K\right), \\
\pi_{\phi} & \equiv \frac{\partial S}{\partial \dot{\phi}}=\frac{1}{2 \kappa^{2}} \dot{\phi}
\end{aligned}
$$

These canonical momenta enable us to reexpress the action as the first-order form

$$
S=\int d^{4} x d y \sqrt{g}\left(\pi_{\mu \nu} \dot{\gamma}^{\mu \nu}+\pi_{\phi} \dot{\phi}-N \mathcal{H}\right)
$$

where the Hamiltonian density is given by 


$$
\mathcal{H}=2 \kappa^{2}\left(\gamma^{\mu \rho} \gamma^{\nu \sigma} \pi_{\mu \nu} \pi_{\rho \sigma}-\frac{1}{3} \pi^{2}+\frac{1}{2} \pi_{\phi}^{2}\right)-\frac{\Lambda}{\kappa^{2}},
$$

with $\pi=\gamma^{\mu \nu} \pi_{\mu \nu}$. The variation of this action with respect to the lapse function leads to the Hamiltonian constraint, $\mathcal{H}=0$. This Hamiltonian is a generator of the translation in the $y$-direction. All solutions connected by this transformation are gauge equivalent.

After imposing the Hamiltonian constraint, the variation of the action finally results in the variation of the boundary action

$$
\delta S_{B}=\int_{\partial \mathcal{M}} d^{4} x \sqrt{\gamma}\left(\pi_{\mu \nu} \delta \gamma^{\mu \nu}+\pi_{\phi} \delta \phi\right),
$$

where all variables are defined at the boundary. In the Hamilton-Jacobi formalism, the canonical momenta are defined as

$$
\pi_{\mu \nu}=\frac{1}{\sqrt{\gamma}} \frac{\delta S_{B}}{\delta \gamma^{\mu \nu}} \quad \text { and } \quad \pi_{\phi}=\frac{1}{\sqrt{\gamma}} \frac{\delta S_{B}}{\delta \phi}
$$

According to the AdS/CFT correspondence, the above boundary action is identified with the generating functional of the dual QFT. To see this relation explicitly and to relate the bulk equations with the RG equations correctly, we resolve the following two issues. First, we have to clarify how the radial position of the boundary is related to the RG scale [48-50]. Second, the above boundary action is an unrenormalized generating functional because we did not get rid of UV divergences yet. Therefore, we have to renormalize it by adding appropriate counterterms.

Although the RG flow can vary the coupling constants including the metric, it does not change the background geometry where the QFT is defined. On the dual gravity side, this implies that the boundary spacetime described by $d s_{B}^{2}=\gamma_{\mu \nu} d x^{\mu} d x^{\nu}$ must be invariant under the scale transformation. Since the metric component in (3.15) scales by $e^{A(\bar{y})} \rightarrow e^{\sigma} e^{A(\bar{y})}$ under $x \rightarrow e^{-\sigma} x$ or $\mu \rightarrow e^{\sigma} \mu$, we can associate the RG scale $\mu$ with the metric component at the boundary, $y=\bar{y}$,

$$
\mu=\frac{e^{A(\bar{y})}}{R}
$$

where $A(\bar{y})$ indicates the value of $A$ at the boundary. This relation describes how the RG scale changes when the boundary moves in the radial direction. From now on, we concentrate on the dual QFT defined at the boundary, so we drop out the bar symbol. For $\eta= \pm 1$, the RG scale is related to the radial position of the boundary

$$
\mu=\frac{e^{y / R}}{R}\left(1-\frac{\phi_{1}^{2}}{96} e^{-8 y / R}\right)^{1 / 4} .
$$

To describe the RG flow, we have to specify counterterms to remove the UV divergences [51,52]. In the renormalization procedure, there may exist several different counterterms which have the same UV divergences [51]. Although the regular terms do not give any contribution to the renormalization, they significantly affect on the RG flow in the intermediate energy scale. This regular contribution is crucially related to the quantum correction and seriously modify the IR physics. Therefore, it is important to determine the counterterms correctly to understand the RG flow and its IR physics. The UV divergence of the above boundary action appears due to the invariant integral measure at a UV cutoff $(\bar{y} \rightarrow \infty)$

$$
\int d^{4} x \sqrt{\gamma} \sim \int d^{4} x e^{4 \bar{y} / R}
$$

On the other hand, the integrands of the boundary action in the asymptotic region behave as

$$
\begin{aligned}
\pi_{\mu \nu} \gamma^{\mu \nu} & \sim \dot{A} \sim \frac{1}{R}, \\
\pi_{\phi} \phi & \sim e^{-4 \bar{y} / R} .
\end{aligned}
$$

As shown in these asymptotic behaviors, the gravity part, $\int d^{4} x \sqrt{\gamma} \pi_{\mu \nu} \gamma^{\mu \nu}$, gives rise to a UV divergence proportional to $e^{4 \bar{y} / R}$, whereas the scalar field part, $\int d^{4} x \sqrt{\gamma} \pi_{\phi} \phi$, does not make any additional UV divergence. Therefore, we need the counterterm which cancels only the divergence of the gravity part. In other words, since the marginal deformation does not make any additional UV divergence, it is sufficient to exploit the same counterterm used in the undeformed CFT [45]

$$
S_{c t}=-\frac{1}{2 \kappa^{2}} \int d^{4} x \sqrt{\gamma} \mathcal{L}_{c t},
$$

with

$$
\mathcal{L}_{c t}=\frac{6}{R} .
$$

Then, the renormalized action is given by

$$
\Gamma\left[\gamma_{\mu \nu}, \phi ; \bar{y}\right]=S_{B}-S_{c t} .
$$

This renormalized boundary action is finite and corresponds to the renormalized generating functional of the dual QFT.

The UV cutoff we introduced is artificial, so that the renormalized action must be independent of the artificial UV cutoff. The scale independence of the renormalized action leads to the following RG equation 


$$
0=\mu \frac{\partial \Gamma}{\partial \mu}+\frac{\partial \gamma^{\mu \nu}}{\partial \log \mu} \frac{\partial \Gamma}{\partial \gamma^{\mu \nu}}+\frac{\partial \phi}{\partial \log \mu} \frac{\partial \Gamma}{\partial \phi},
$$

where $\mu$ is the RG scale satisfying (3.24). Due to the following relation

$$
\frac{d \gamma^{\mu \nu}}{d \log \mu}=-2 \gamma^{\mu \nu}
$$

we further rewrite the above RG equation as the usual form

$$
0=\frac{\mu}{\sqrt{\gamma}} \frac{\partial \Gamma}{\partial \mu}+\gamma^{\mu \nu}\left\langle T_{\mu \nu}\right\rangle+\beta_{\phi}\langle O\rangle,
$$

with the following definitions

$$
\begin{aligned}
\beta_{\phi} & \equiv \frac{\partial \phi}{\partial \log \mu}, \\
\left\langle T_{\mu \nu}\right\rangle & \equiv-\frac{2}{\sqrt{\gamma}} \frac{\partial \Gamma}{\partial \gamma^{\mu \nu}}=-\left(2 \pi_{\mu \nu}-\frac{1}{2 \kappa^{2}} \gamma_{\mu \nu} \mathcal{L}_{c t}\right), \\
\langle O\rangle & \equiv \frac{1}{\sqrt{\gamma}} \frac{\delta \Gamma}{\delta \phi}=\pi_{\phi}+\frac{1}{2 \kappa^{2}} \frac{\partial \mathcal{L}_{c t}}{\partial \phi} .
\end{aligned}
$$

This is the same as the generalized RG equation of a QFT in (2.5). Above, $\phi$ indicates the boundary value of the bulk scalar field. Under the identification of $\phi$ with the dimensionless coupling, the VEV of the deformation operator is determined as a derivative of the generating functional with respect to the coupling constant, as shown in (3.35) and (3.36). In this case, the VEV is usually determined as a function of coupling constants.

\section{B. RG flow triggered by a marginal operator}

When we describe the holographic RG flow, it is more convenient to introduce a superpotential. To see more details, we return to the bulk equations of motion. Since the bulk equations, (3.7), (3.8), and (3.9), are depending only on $\dot{A}$ and $\ddot{A}$, we can introduce a superpotential satisfying $[13,46,53-57]$

$$
W(\phi)=6 \dot{A} .
$$

Then, the bulk equations reduce to two first-order differential equations

$$
\begin{gathered}
2 \Lambda=\frac{1}{2}\left(\frac{\partial W}{\partial \phi}\right)^{2}-\frac{1}{3} W^{2}, \\
\dot{\phi}=-\frac{\partial W}{\partial \phi} .
\end{gathered}
$$

Here the first equation corresponds to the Hamiltonian constraint which determines the superpotential as a function of $\phi$. Using this superpotential, we rewrite the $\mathrm{RG}$ equation in terms of the superpotential

$$
0=\frac{\mu}{\sqrt{\gamma}} \frac{\partial \Gamma}{\partial \mu}+\gamma^{\mu \nu}\left\langle T_{\mu \nu}\right\rangle+\beta_{\phi}\langle O\rangle,
$$

with

$$
\begin{aligned}
\beta_{\phi} & =-\frac{6}{W} \frac{\partial W}{\partial \phi}, \\
\left\langle T_{\mu \nu}\right\rangle & =\frac{1}{\kappa^{2}}\left(K_{\mu \nu}-\gamma_{\mu \nu} K\right)-\frac{3}{\kappa^{2} R} \gamma_{\mu \nu}, \\
\langle O\rangle & =\frac{1}{2 \kappa^{2}} \frac{\partial W}{\partial \phi} .
\end{aligned}
$$

For a marginal deformation, $\partial \mathcal{L}_{c t} / \partial \phi$ automatically vanishes because $\mathcal{L}_{c t}$ is independent of $\phi$, as shown in (3.29).

\section{Marginally relevant or irrelevant deformation}

The Hamiltonian constraint (3.38) allows two different types of solutions. The first one is given by $[15,16,46,47]$

$$
W=\frac{6}{R} \cosh \left(\sqrt{\frac{2}{3}}\left(\phi-\phi_{0}\right)\right) .
$$

After substituting the superpotential into (3.39) and solving it, we reobtain the previous gravity solutions, (3.10) and (3.11) with $\eta= \pm 1$. From the profile of the bulk scalar field (3.10), we can easily read off the corresponding $\beta$-function in the UV region

$$
\beta_{\phi}=\frac{\eta \phi_{1}}{\mu^{4}}+\mathcal{O}\left(\frac{1}{\mu^{12}}\right) .
$$

Recalling that the $\beta$-function of the marginal operator vanishes at the classical level, the above nonvanishing $\beta$-function comes thoroughly from the quantum effect. This fact indicates that the deformation operator is marginally relevant $\left(\beta_{\phi}<0\right)$ for $\eta=-1$ or marginally irrelevant $\left(\beta_{\phi}>0\right)$ for $\eta=1$, see Fig. 1 . On the other hand, if we identify the bulk scalar field with the inverse of a coupling constant as will be seen in the next section, the deformation becomes marginally relevant for $\eta=1$ and marginally irrelevant for $\eta=-1$. We see from (3.36) that the VEV of the deformation operator reduces to

$$
\langle O\rangle=\frac{\sqrt{6}}{\kappa^{2} R} \sinh \left(\sqrt{\frac{2}{3}}\left(\phi-\phi_{0}\right)\right),
$$

which is a function of the coupling constant $\phi$, as mentioned before. In the UV region, as will be shown in the next section, the VEV is proportional to $\phi_{1} \mu^{-4}$ at leading order. This is the form expected by the RG flow of a 


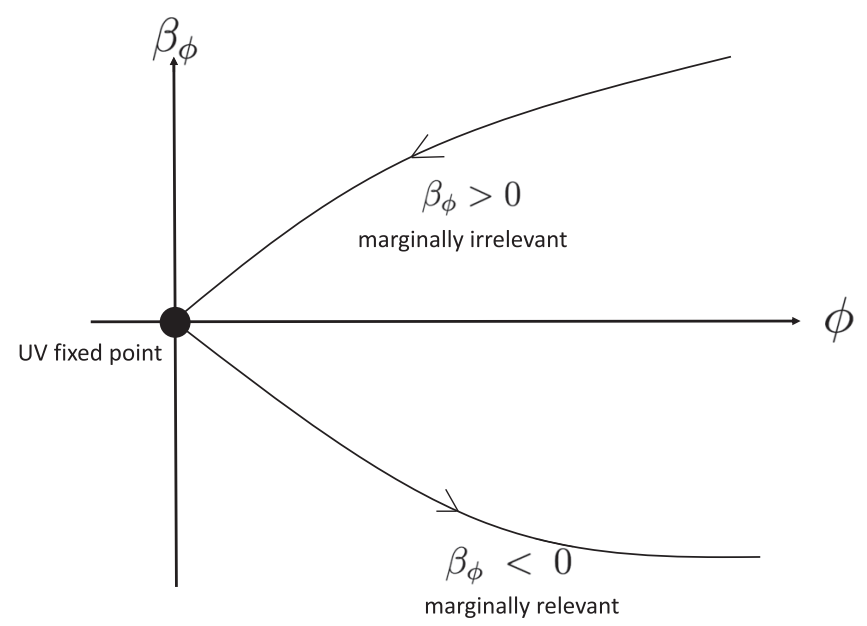

FIG. 1. The RG flows caused by marginally relevant and irrelevant operators.

QFT. More precisely, the VEV scales by $\mu^{-4}$ for $\Delta=4$, as mentioned in (2.2). In addition, the second integral constant $\phi_{1}$ determines the VEV in the UV limit. This fact indicates that the new identification used in this work is consistent with the UV identification of Ref. [2-4] at least in the UV region. Unlike the UV identification, however, the new identification allows us to describe the change of the coupling constant along RG flow.

\section{Truly marginal deformation}

There exists another superpotential satisfying the Hamiltonian constraint. The second superpotential is given by

$$
W=\frac{6}{R}
$$

which corresponds to the case of $\phi=\phi_{0}$ in (3.44). This superpotential reproduces the trivial solution, (3.10) with $\eta=0$. Plugging this superpotential into (3.39) and (3.37), we finally reobtain the trivial solution in (3.13). In this case, the coupling constant $\phi=0$ of the undeformed CFT changes into $\phi=\phi_{0}$ of the deformed CFT. However, the $\beta$-function automatically vanishes. This is a typical feature of the truly marginal deformation. A truly marginal operator changes a CFT into another CFT by shifting the coupling constant without generating a nontrivial RG flow. The dual geometry of the truly marginal deformation is again given by an AdS space, as expected.

\section{HOLOGRAPHIC DUAL OF THE GLUON CONDENSATION}

Several distinct condensations occur in QCD. They are usually associated with a certain spontaneous symmetry breaking and responsible for the mass of hadrons. Due to the Lorentz invariance, the condensation should be a
Lorentz scalar and has vanishing charges under various global and local symmetries. The well-known condensations in QCD are the chiral and gluon condensations. The chiral condensation is the condensate of two fermions with breaking the chiral symmetry spontaneously and yields a large effective mass to quarks and most hadrons. The other example is the gluon condensation. For a four-dimensional Yang-Mills theory, the kinetic term of the gauge field is given by

$$
S_{\mathrm{YM}}=-\frac{1}{4 g_{\mathrm{YM}}^{2}} \int d^{4} x \sqrt{\gamma} \operatorname{Tr} F^{2}
$$

In this case, since the Yang-Mills coupling constant is classically marginal, the classical $\beta$-function automatically vanishes. However, the one-loop quantum correction generates a nonvanishing $\beta$-function $[5,58,59]$. In general, QCD contains many ingredients and condensations, so that a dual gravity theory also has many bulk fields corresponding to such QCD contents. Unfortunately, a dual gravity theory of QCD is still unknown. If we take into account other relevant deformations of QCD, there may be a mixing of marginal and relevant deformations. Such mixing of deformation makes the theory have a more rich structure and allows other interesting IR physics. We leave the study on this issue as future works. In the present work, we concentrate only on the gluon condensation for a marginal deformation, ignoring the effects of other QCD contents. Then, the dual gravity of the gluon condensation is well approximated by the previous Einstein-scalar gravity. This study is sufficient to clarify the effect of a marginal deformation discussed before.

When QCD deforms by the gluon condensation, we are able to regard $\phi=1 /\left(4 g_{\mathrm{YM}}^{2}\right)$ as a coupling constant and $G=-\operatorname{Tr} F^{2}$ as a deformation operator whose vacuum expectation value (VEV) corresponds to the gluon condensation. Although it is not clear whether the gluon condensation is associated with the known phase change, there are many indications for the nonvanishing gluon condensation in lattice QCD simulations. The gluon condensation may be partly responsible for masses of hadrons and leads to a nontrivial RG flow closely related to the trace anomaly. The lattice QCD simulations expects that the oneloop quantum correction in the UV region leads to the following trace anomaly $[21,22]$

$$
\left\langle T^{\mu}{ }_{\mu}\right\rangle=-\frac{N_{c}}{8 \pi} \frac{\beta_{\lambda}}{\lambda^{2}}\langle G\rangle,
$$

where $N_{c}$ indicates the rank of the gauge group and $\beta_{\lambda}$ means a $\beta$-function of the 't Hooft coupling constant $\lambda$. In general, the condensation plays a crucial role in nonperturbative phenomena. To understand such nonperturbative features, the AdS/CFT correspondence may be helpful. The effect of the gluon condensation on the trace anomaly can be understood by using the holographic RG 
flow discussed before. Although the gravity model we considered is too simple to study IR physics, the present model is still useful to account for the UV physics because it gives rise to the leading behavior of the marginal deformation at least in the UV region.

Now, we specify the parameters of the gravity theory in terms of those of the dual QFT. For applying the AdS/CFT correspondence, we define the 't Hooft coupling constant as $\lambda=N_{c} g_{\mathrm{YM}}^{2}$ and take the double scaling limit where $N_{c} \rightarrow \infty$ and $g_{\mathrm{YM}}^{2} \rightarrow 0$ with a fixed $\lambda$. Despite an infinite rank of the gauge group, the AdS/CFT correspondence still has an advantage in catching the important feature of the nonperturbative RG flow. If we identify the VEV of the dual operator with the gluon condensation, $\langle G\rangle$, the bulk scalar field is associated with the 't Hooft coupling constant

$$
\phi=\frac{N_{c}}{4 \lambda} .
$$

In the large 't Hooft coupling limit $(\lambda \gg 1)$, the leading behavior of $\phi$ is proportional to $1 / \mu^{4}$ in (3.10). This implies that the 't Hooft coupling constant is related to the RG scale by $\lambda \sim \mu^{4}$ in the UV region ( $\left.\mu \gg 1\right)$. The $\beta$-function (3.45) derived from the bulk scalar field can be reexpressed as that of the 't Hooft coupling constant

$$
\beta_{\phi}=-\frac{N_{c}}{4} \frac{\beta_{\lambda}}{\lambda^{2}}
$$

When we identify the bulk scalar field with the inverse of the 't Hooft coupling constant in (4.3), we have to take $\eta=1$ rather than $\eta=-1$ for describing a marginally relevant deformation. For example, if the rank of the gauge group $N_{c}$ is sufficiently larger than the number of other matter fields, $\beta_{\lambda}$ has a negative value representing a marginally relevant deformation. In (4.4), the negative $\beta_{\lambda}$ enforces a positive $\beta_{\phi}$ which is the same as choosing $\eta=+1$ in (3.45). This is also consistent with our previous prescription that, when the bulk field is identified with the inverse of the coupling constant, $\eta=+1$ describes a marginally relevant deformation.

From the holographic RG flow description studied before, the $\beta$-function and the gluon condensation in the $\mathrm{UV}$ region are given by functions of the RG scale

$$
\begin{aligned}
\beta_{\phi} & =\frac{\phi_{1}}{R^{4}} \frac{1}{\mu^{4}}-\frac{\phi_{1}^{3}}{48 R^{12}} \frac{1}{\mu^{12}}+\mathcal{O}\left(\mu^{-20}\right), \\
\langle G\rangle & =-\frac{\phi_{1}}{2 \kappa^{2} R^{5}} \frac{1}{\mu^{4}}+\mathcal{O}\left(\mu^{-28}\right) .
\end{aligned}
$$

Here the $\beta_{\lambda}$ and $\langle G\rangle$ are also represented as functions of the 't Hooft coupling constant, for example, $\beta_{\lambda} \sim-\lambda$ and $\langle G\rangle \sim-1 / \lambda$ in the UV region. The leading behavior of the gluon condensation, $\langle G\rangle \sim \mu^{-4}$, is the form expected by the classical dimension counting in (2.2). This result shows that the gluon condensation rapidly suppresses as the 't Hooft coupling constant increases. From (3.36), moreover, we show that the trace anomaly has the following RG scale dependence

$$
\left\langle T_{\mu}^{\mu}\right\rangle=-\frac{\phi_{1}^{2}}{4 \kappa^{2} R^{9}} \frac{1}{\mu^{8}}+\frac{\phi_{1}^{4}}{384 \kappa^{2} R^{17}} \frac{1}{\mu^{16}}+\mathcal{O}\left(\mu^{-24}\right) .
$$

Reexpressing the trace anomaly in terms of the coupling constant and gluon condensation, we finally find that the trace anomaly is closely related to the gluon condensation

$$
\left\langle T^{\mu}{ }_{\mu}\right\rangle=-\frac{N_{c}}{8} \frac{\beta_{\lambda}}{\lambda^{2}}\langle G\rangle+\mathcal{O}\left(\lambda^{-4}\right) .
$$

Here, the leading contribution satisfies the trace anomaly expected in the lattice QCD [21,22]. Intriguingly, this holographic result shows that the trace anomaly of the lattice QCD (4.2) is valid only at the one-loop level. At the higher-loop level, there exists an additional contribution at the $\lambda^{-4}$ order. As a result, the higher-order correction modifies the one-loop trace anomaly. Lastly, the holographic description of the gluon condensation leads to the expected behavior of the RG flow. For example, the gluon condensation and the trace anomaly rapidly suppress in the UV region and then the conformal symmetry is restored at the UV fixed point.

\section{DISCUSSION}

In this work, we studied the holographic RG flow of a CFT deformed by a marginal operator. We discussed how we can understand the RG flow of a boundary QFT in terms of the Hamilton-Jacobi formulation on the dual gravity side. At the classical level, a marginal operator does not change the CFT. The quantum effect, however, can lead to nontrivial modification of the CFT along the RG flow. Using the holographic description, we studied how the quantum effect of the marginal deformation modifies the $\beta$-function and the VEV of the operator. Furthermore, we compared this holographic result with the gluon condensation known in QCD.

There were several distinct prescriptions to realize the nonperturbative RG flow of the QFT on the dual gravity side. In the present work, we exploited the Hamilton-Jacobi formalism which allows us to rewrite the gravity equations as the first-order differential equations. The RG equations are generally given by the first-order differential equations, so that the Hamilton-Jacobi formulation is useful to understand the RG flow of the dual QFT. When we applied the Hamilton-Jacobi formulation, it suffers from the UV divergences similar to those appearing in the QFT renormalization. We discussed the counterterms, which get rid of the UV divergences, and then find the finite boundary action corresponding to the generating functional of the dual QFT. 
After the holographic renormalization, we explicitly showed that the quantum correction modifies a classically marginal operator into one of the truly marginal, marginally relevant and marginally irrelevant operators at the quantum level. More precisely, the quantum correction gives rise to a nonvanishing $\beta$-function for marginally relevant and irrelevant deformations. If we focus on the marginally relevant deformation, the undeformed theory at the UV fixed point becomes unstable. Thus, the UV CFT flows to a new IR theory. In this case, the other quantities like the stress tensor and VEV of the operator also vary along the RG flow. We explicitly calculated the RG scale dependence of those quantities near the UV fixed point. We also showed that the holographic RG flow reproduces the known trace anomaly of the gluon condensation [21,22]. Intriguingly, the holographic RG flow further shows that the trace anomaly formula of the lattice QCD is valid only at the $\lambda^{-2}$ order.

In the present work, the Einstein-scalar gravity we considered has no well defined IR geometry because of the existence of a singularity at $z^{4}=4 \sqrt{6} / \phi_{1}$. This means that the dual QFT of the present model is IR incomplete, so that the end of the RG flow is not manifest. This means that the present model is not valid in the IR region. Nevertheless, the results we obtained in this work are still valid in the UV region because they corresponds to the leading contribution. To study the IR physics further, we have to consider a more general gravity theory which allows a well-defined IR fixed point. For example, we can take into account a scalar potential with higher-order terms

$$
V(\phi)=\sum_{n \geq 3} a_{n} \phi^{n}
$$

For a relevant deformation, this potential crucially modifies IR physics and can allow an IR fixed point. Despite this fact, its contribution in the UV region becomes subdominant. Due to this reason, in this work we concentrate on the UV behavior of the RG flow. Nevertheless, it is still important to take into account higher-order terms to understand interesting IR phenomena. We hope to report more results on this issue in future works.

\section{ACKNOWLEDGMENTS}

This work was supported by the National Research Foundation of Korea (NRF) grant funded by the Korea government (MSIT) (No. NRF-2019R1A2C1006639).
[1] J. M. Maldacena, Int. J. Theor. Phys. 38, 1113 (1999); Adv. Theor. Math. Phys. 2, 231 (1998).

[2] S. Gubser, I. R. Klebanov, and A. M. Polyakov, Phys. Lett. B 428, 105 (1998).

[3] E. Witten, Adv. Theor. Math. Phys. 2, 253 (1998).

[4] E. Witten, Adv. Theor. Math. Phys. 2, 505 (1998).

[5] K. Wilson and J. B. Kogut, Phys. Rep. 12, 75 (1974).

[6] M. Henningson and K. Skenderis, J. High Energy Phys. 07 (1998) 023.

[7] M. Henningson and K. Skenderis, Fortschr. Phys. 48, 125 (2000).

[8] D. Z. Freedman, S. D. Mathur, A. Matusis, and L. Rastelli, Nucl. Phys. B546, 96 (1999).

[9] S. S. Gubser, Phys. Rev. D 63, 084017 (2001).

[10] J. de Boer, E. P. Verlinde, and H. L. Verlinde, J. High Energy Phys. 08 (2000) 003.

[11] E. P. Verlinde and H. L. Verlinde, J. High Energy Phys. 05 (2000) 034.

[12] J. de Boer, Fortschr. Phys. 49, 339 (2001).

[13] K. Skenderis and P. K. Townsend, Phys. Lett. B 468, 46 (1999).

[14] J. Polchinski and J. Sully, J. High Energy Phys. 10 (2011) 059.

[15] I. Papadimitriou, J. High Energy Phys. 11 (2010) 014.

[16] I. Papadimitriou and K. Skenderis, IRMA Lect. Math. Theor. Phys. 8, 73 (2005).

[17] K. Skenderis, Classical Quantum Gravity 19, 5849 (2002).
[18] I. Heemskerk and J. Polchinski, J. High Energy Phys. 06 (2011) 031.

[19] J. Erdmenger, C. M. Melby-Thompson, and C. Northe, J. High Energy Phys. 05 (2020) 075.

[20] B.-H. Lee, S. Nam, and C. Park, J. Korean Phys. Soc. 70, 34 (2017).

[21] A. Di Giacomo and G. Rossi, Phys. Lett. 100B, 481 (1981).

[22] R. C. Trinchero, Int. J. Mod. Phys. A 29, 1450131 (2014).

[23] R. B. Mann, Classical Quantum Gravity 14, L109 (1997).

[24] D. R. Brill, J. Louko, and P. Peldan, Phys. Rev. D 56, 3600 (1997).

[25] L. Vanzo, Phys. Rev. D 56, 6475 (1997).

[26] R. Emparan, Phys. Lett. B 432, 74 (1998).

[27] D. Birmingham, Classical Quantum Gravity 16, 1197 (1999).

[28] A. Chamblin, R. Emparan, C. V. Johnson, and R. C. Myers, Phys. Rev. D 59, 064010 (1999).

[29] R. Emparan, C. V. Johnson, and R. C. Myers, Phys. Rev. D 60, 104001 (1999).

[30] S. Hawking, C. Hunter, and D. N. Page, Phys. Rev. D 59, 044033 (1999).

[31] N. Lashkari, M. B. McDermott, and M. Van Raamsdonk, J. High Energy Phys. 04 (2014) 195.

[32] B. Swingle and M. Van Raamsdonk, arXiv:1405.2933.

[33] X. Dong, D. Harlow, and A. C. Wall, Phys. Rev. Lett. 117, 021601 (2016).

[34] S. Ryu and T. Takayanagi, Phys. Rev. Lett. 96, 181602 (2006). 
[35] S. Ryu and T. Takayanagi, J. High Energy Phys. 08 (2006) 045.

[36] T. Nishioka, S. Ryu, and T. Takayanagi, J. Phys. A 42, 504008 (2009).

[37] T. Takayanagi, Classical Quantum Gravity 29, 153001 (2012).

[38] R. Narayanan, C. Park, and Y.-L. Zhang, Phys. Rev. D 99, 046019 (2019).

[39] C. Park, D. Ro, and J. Hun Lee, J. High Energy Phys. 11 (2018) 165.

[40] C. Park and J. Hun Lee, Phys. Rev. D 101, 086008 (2020).

[41] K.-S. Kim and C. Park, Phys. Rev. D 95, 106007 (2017).

[42] T. J. Hollowood, arXiv:0909.0859.

[43] D. Friedan, Phys. Rev. Lett. 45, 1057 (1980).

[44] D. H. Friedan, Ann. Phys. (N.Y.) 163, 318 (1985).

[45] V. Balasubramanian and P. Kraus, Commun. Math. Phys. 208, 413 (1999).

[46] C. Csaki and M. Reece, J. High Energy Phys. 05 (2007) 062.
[47] Y. Kim, B.-H. Lee, C. Park, and S.-J. Sin, J. High Energy Phys. 09 (2007) 105.

[48] K.-S. Kim, M. Park, J. Cho, and C. Park, Phys. Rev. D 96, 086015 (2017).

[49] K.-S. Kim and C. Park, Phys. Rev. D 93, 121702 (2016).

[50] K.-S. Kim, S. B. Chung, C. Park, and J.-H. Han, Phys. Rev. D 99, 105012 (2019).

[51] C. Park, Phys. Rev. D 89, 066003 (2014).

[52] C. Park, Int. J. Mod. Phys. A 29, 1430049 (2014).

[53] D. Freedman, S. Gubser, K. Pilch, and N. Warner, Adv. Theor. Math. Phys. 3, 363 (1999).

[54] O. DeWolfe, D. Freedman, S. Gubser, and A. Karch, Phys. Rev. D 62, 046008 (2000).

[55] C. Csaki, J. Erlich, C. Grojean, and T. J. Hollowood, Nucl. Phys. B584, 359 (2000).

[56] S. S. Gubser, arXiv:hep-th/9902155.

[57] A. Kehagias and K. Sfetsos, Phys. Lett. B 454, 270 (1999).

[58] D. Gross and F. Wilczek, Phys. Rev. D 8, 3633 (1973).

[59] G. 't Hooft, Nucl. Phys. B, Proc. Suppl. 74, 413 (1999). 